\title{
Characterisation of small targets in a maritime environment by means of laser range profiling
}

\author{
Robin Schoemaker \& Koen Benoist \\ TNO Defence, Security and Safety, P.O. Box 96864, 2509 JG, The Hague, The Netherlands \\ Tel: +31 88866 4181, Fax: +31 88866 6575, E-mail: robin.schoemaker@tno.nl
}

\begin{abstract}
Potential asymmetric threats at short range in complex environments need to be identified quickly during coastal operations. Laser range profiling is a technology that has the potential to shorten the OODA loop (Orient, Observe, Detect, Act) by performing automatic characterisation of targets at large distance. The advantages of non-cooperative target recognition with range profiles are: (a) a relatively short time on target is required, (b) the detection range is longer than in the case of passive observation technologies such as IRST, and (c) characterisation of range profiles is possible at any aspect angle. However, the shape of a range profile depends strongly on aspect angle. This means that a large data set is necessary of all expected targets with reference profiles on a very dense aspect angle grid. Analysis of laser range profiles can be done by comparing the measured profile with a database of laser range profiles obtained from 3D models of possible targets. An alternative is the use of a profile database from one or several measurement campaigns. A prerequisite for this is the availability of enough measured profiles of the appropriate targets, for many aspect angles. Comparison of measured laser range profiles with a reference database can be performed using, e.g., formal statistical correlation techniques or histogram dissimilarity techniques.

In this work, a field trial has been conducted to validate the concept of identification by using a laser range profiling system with a high bandwidth receiver and short laser pulses. The field trial aimed at characterization of sea-surface targets in a coastal/harbour environment. The targets ranged from pleasure boats like sailing boats, jet skis, and speed boats to professional vessels like barges, cabin boats, and military vessels, all ranging from 3 to 30 meters in length. We focus on (a) the use of a reference database generated via 3D target models, and (b) the use of a reference database of measured laser range profiles. A variety of histogram dissimilarity measures was examined in order to enable fast and reliable classification algorithms.
\end{abstract}

Keywords: Laser range profiling, target characterisation, small maritime targets.

\section{INTRODUCTION}

The basis for acquiring target knowledge with sensors is the detection and characterisation of persons and objects in an observation sector. After detection of the target, the characterisation levels, which run hierarchically through discrimination, categorisation, recognition, and identification, depend on the amount of available information in the sensed data and the information requirements for the different levels. Hardware, software, and the environment in which the sensor/target combination operates, determine the achievable level (Figure 2.1). Furthermore, characterisation methods of the measured laser range profiles were investigated.

In this paper 'target characterisation' is used as a general term for achieving the best target knowledge with active imaging techniques. A laser system operating with extremely short wavelength EM transmissions has the potential to end up in the recognition and identification phase.

Laser range profiling (LRP) is a measurement technique to characterise an object by the shape of laser pulses that reflect off the object's surface. The setup consists of a laser that emits short pulses, and a fast detector to detect the reflected energy. Features of the object that turn up in the reflection signal can be compared with a priori information stored in a database, or can be analysed on uniqueness using artificial neural networks. As the wavelength of the laser light is extremely small, high-resolution details can be distinguished at great distance.

Laser Radar Technology and Applications XVI, edited by Monte D. Turner, Gary W. Kamerman, Proc. of SPIE Vol. 8037, 803705 - @ 2011 SPIE · CCC code: 0277-786X/11/\$18 · doi: 10.1117/12.884575 


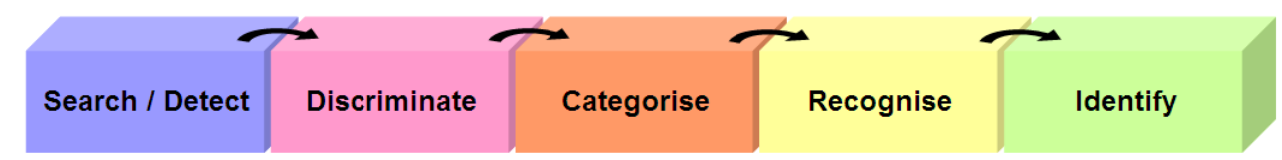

Figure 2.1: Five phases of sensor functionality in the target characterisation procedure. From left to right the characterisation provides more information.

Three questions have been opted regarding laser range profiling in relation to small target characterisation with real and simulated data. They form the basis of this paper: (i) Which numerical methods are suitable for target identification? (ii) Are 3D models convenient to generate a database for laser range profiling? (iii) Which range resolution is feasible for target characterisation and which targets are identifiable for a certain resolution?

The paper is arranged as follows. Section 2 starts with a short introduction on the rationale of the laser range profiling methodology. TNO's laser range profiler, its make-up and its operation, is explored in Section 3 as is a short discussion on the experimental set-up in a measurement trial. Hardware and data require dedicated software to deliver the target range profiles. So, the software methodology is discussed in Section 4, as well are the results from the characterisation methods used. Section 5 comes with conclusions and addresses the above questions as such.

\section{LASER RANGE PROFILING: RATIONALE}

Laser range profiling originates from its radar equivalent called radar range profiling [1-4]. The basic idea of radar range profiling is that the geometry of the target or the moving parts of the target imposes features in the reflected radar signal that are typical for the object. These features may then be used to classify the target when the measured profile is compared with simulated profiles from an a priori 3D model database.

\subsection{Radar}

Radar range profiling varies as function of the aspect angle. Small variations of this angle can have a profound effect on the range profile. Since the surface of the aircraft is smooth with respect to radar waves, specular reflection dominates. This specular reflection is very sensitive to aspect angles as it is comparable to mirror reflections. Another is related to speckle effects. If two point scatterers in the same range cell are located at opposite edges of the aircraft, then a slight rotation of the aircraft can cause the radar return interference from the two scatterers to be destructive instead of constructive. If a flat part of the aircraft happens to be pointed perpendicular to the radar line of sight, a large radar crosssection/reflection from this part is observed. Such a flash is highly aspect angle dependent. Specular reflections may be utilised for target characterisation, if the dynamic behaviour or a sequence of range profiles is considered. If not, specular reflection causes the reflection from other parts of the aircraft to be masked. In general, target characterisation based on high resolution range (HRR) radar profiles requires a larger signal to noise ratio with respect to the total amount of energy needed for detection. In fact, for detection it is only required that the reflection from an aircraft exceeds a certain threshold. The next step, characterisation, requires different amplitudes from scatterers in the HRR profile, since these scatterers compose the identity of the aircraft. Implementations of HRR techniques in radar systems require that those radars be wideband and coherent. The most important requirements for non-cooperative target recognition relate to the geometrical resolutions (range resolutions in HRR). Resolution requirements impose radar system bandwidth requirements, and bandwidth is a key parameter which is usually limited by the transmitter or antenna technology for a given radar design.

Obviously, radar range profiling has some disadvantages. It is therefore not surprising that the same techniques have been explored in the optical domain.

\subsection{Laser}

Probably the earliest application of lasers in a military context concerns the laser range finder. The laser range finder measures the distance from the platform to the target by measuring the time it takes a pulse to travel back and forth between platform and target (time-of-flight/range measurement principle). A laser range profiler, such as the one used for this research, does not measure the first, second or last echo return, but instead measures the complete shape of the return laser pulse. This so-called laser range profile will depend on the angle between the laser beam and the target. A laser range profiler has several attractive properties: 
- It has a very high angular resolving power and hence multiple targets can be probed individually. Side lobes, prone to errors for RF wavelengths, are absent.

- It has less specular reflection effects compared to radar since the surface of most parts of the target can be considered rough relative to the wavelength of the laser light.

- Because a laser has a much smaller wavelength and the reflection is mostly diffuse, the returns within a single range interval add up incoherently most of the time and interference from scatterers is absent.

- Because the reflection is mostly diffuse, laser range profilers can be used effectively against radar-stealth craft.

The result is that the laser range profile gives more direct information on the geometry of the target and the profile is less distorted by coherent artefacts. This could make tar-get categorisation, recognition, and identification less complex in comparison to its radar equivalent. This is only meaningful if the measured profile can be compared with other data from similar objects. The latter is often done by comparing measured profiles with simulated profiles from a database. The question then arises on how discriminative these laser range profiles are. Apart from the hardware and environment, the numerical methods, that recover most information from a laser range profiling measurement, are of interest too. In Chapter 5 we discuss the foremost numerical methods that can be used for proper target characterisation.

In Figure 2.2 an example for a simulated high-resolution laser range profile 'measurement' is shown. A 3D model of the F-16 was 'measured' from the front, resulting in a characteristic signature for that specific aspect angle. This signature, one of many for the database, can then be compared with a real measurement of an F-16 for that same aspect angle in a characterisation procedure.
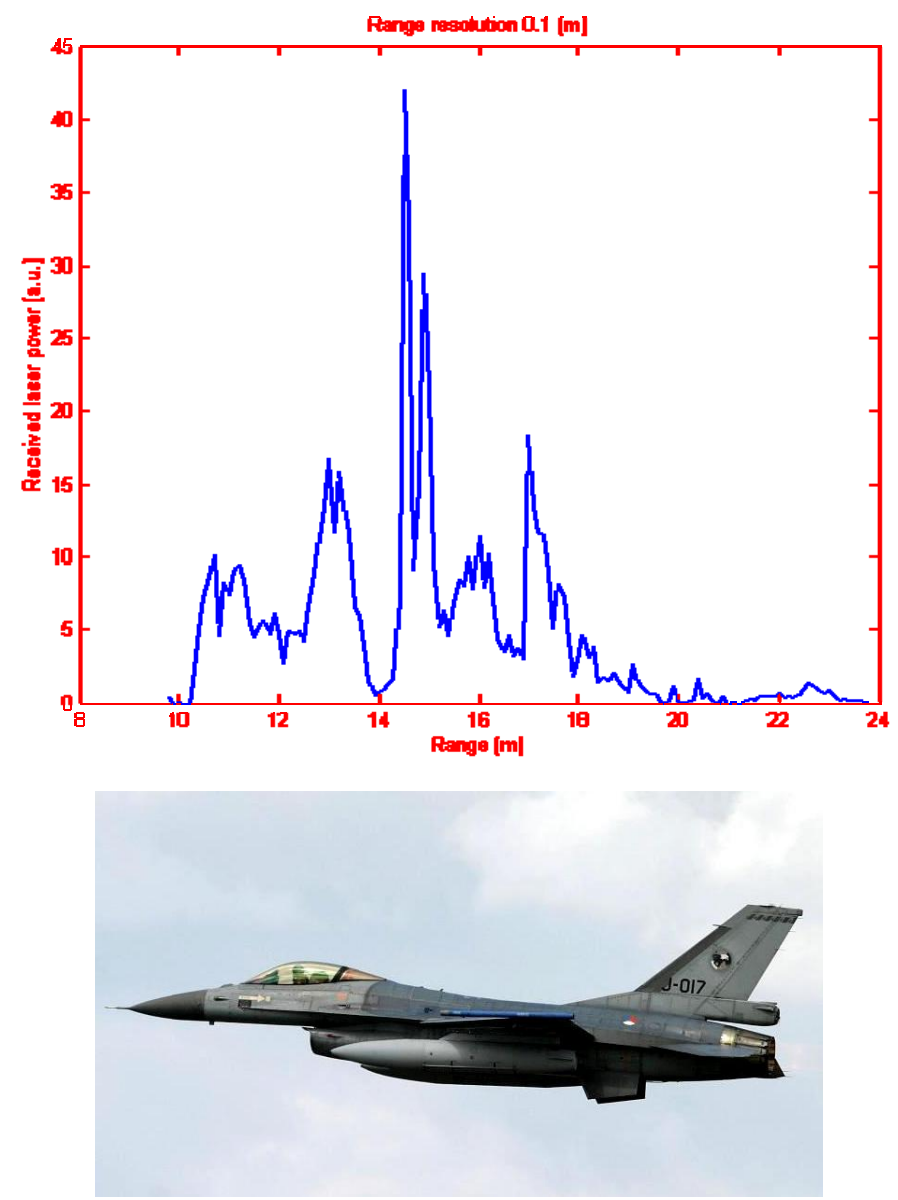
Figure 2.2: Upper panel: Example of a simulated laser range profile of an F16 Fighter Falcon with a range-resolution of 10 $\mathrm{cm}$. The 3D computer model was targeted from the front (left to right) in a measurement simulation. Bottom panel: Photograph of an F16 on the same scale.

\section{LASER RANGE PROFILING: MEASUREMENTS}

\subsection{Laser, detector, optics and operation}

The present TNO laser range profiler (Figure 3.1) puts $60 \mathrm{~mJ}$ in one laser pulse of $5 \mathrm{~ns}$. The pulse repetition frequency (PRF) is $10 \mathrm{~Hz}$. The divergence is approx. $1 \mathrm{mrad}$ at full width at half maximum (FWHM). The eye-safe output wavelength of $1.5 \mu \mathrm{m}$ exits from an Optical Parametric Oscillator crystal (OPO) pumped with a $1.06 \mu \mathrm{m}$ YAG-based laser. In general, a higher pulse energy has the disadvantage that it goes hand in hand with a lower PRF.
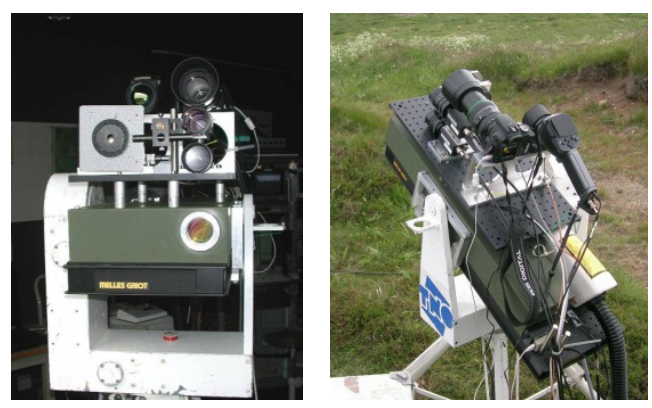

Figure 3.1: The TNO laser range profiler.

In case of a range profiler assembly, a low PRF of $10 \mathrm{~Hz}$ is acceptable because no fast scanning of a large field-of-view (FOV) is required. In case of fast scanning devices, a much higher PRF is necessary in order to fill a wide FOV with overlapping laser pulses.

Commercial-of-the-shelf (COTS) laser systems with multi-mJ laser pulse energies are available, with a typical PRF of $10 \mathrm{~Hz}$ and pulse length of order $10 \mathrm{~ns}$ (for example offered by Spectra-Physics/Newport or Quantel). Pump lasers are also available with COTS OPO systems. The output pulse energy of these systems at $1.5 \mu \mathrm{m}$ is of the order of the TNO LRP assembly, i.e. up to several tens of mJ. Examples of such systems are the Scan Series nanosecond OPO of SpectraPhysics/Newport and the Rainbow OPO of Quantel. State-of-the-art semi-conductor lasers are available for pulse energies up to $400 \mathrm{~mJ}$, and pulse lengths of a few ns.

The present TNO LRP assembly is equipped with a Perkin Elmer InGaAs APD (Ava-lanche Photo Diode) detector, sensitive at $1.5 \mu \mathrm{m}$, with a diameter of $80 \mu \mathrm{m}$ and a bandwidth of $200 \mathrm{MHz}$. This bandwidth is sufficient to record $10 \mathrm{~ns}$ laser pulses. The receiver FOV is determined by the diameter $d$ of the detector and the focal length $f$ of the accompanying optics, according to FOV $=2 \arctan (d / 2 f)$.

At present, two different lenses are available. The first has a diameter of $18 \mathrm{~mm}$ and a focal length of $15 \mathrm{~mm}$, the second has a diameter of $50.8 \mathrm{~mm}$ and a focal length of $50 \mathrm{~mm}$. FOVs for the two lenses in combination with the detector are $5.3 \mathrm{mrad}$ and $1.6 \mathrm{mrad}$, respectively. Note that the lens diameter (or aperture) is relevant for the collected amount of light, but not for the FOV magnitude. The receiver setup may be improved by a higher bandwidth, a larger aperture, and/or a larger detector area.

For the discussed LRP application, however, not all alterations are useful, i.e. a higher detector bandwidth allows for faster measurements, but in the present case a faster detector has no added value. Indeed, since the laser pulse of an average laser system is not shorter than $5 \mathrm{~ns}$, a $200 \mathrm{MHz}$ bandwidth is already sufficient. For faster detectors, the depth resolution is limited by the laser pulse duration. Faster detectors have the additional disadvantage of a higher optical power threshold value.

A larger aperture leads to the collection of more light and therefore to a higher signal-to-noise ratio. However, a larger aperture also implies a larger focal distance (assuming a similar f-number). This means that the FOV becomes smaller. As mentioned before, in the present system two different lenses are available. 
An upgrade of the system can be achieved by installing an Optical Tube Assembly (OTA), which has a large aperture. For example, OTAs with apertures of 8 " (about $20 \mathrm{~cm}$ ) up to 14" (about $35 \mathrm{~cm}$ ) diameters are commercially available. The $f$-numbers are in the order of 10 , so the focal distances are about $2.0 \mathrm{~m}$ and $3.5 \mathrm{~m}$, respectively. These larger focal distances lead to a smaller FOV. Therefore, we have a trade-off between the aperture size and the FOV. The FOV, however, can be enhanced by compensating the larger focal distance with a larger detector. For example, by combining the 8 " OTA with a $2 \mathrm{~mm}$ MCP-PMT (Micro channel plate photomultiplier tube) we arrive at a FOV of $1 \mathrm{mrad}$. Using a 200 $\mu \mathrm{m}$ detector, the FOV would be $100 \mu \mathrm{rad}$, which is much below the laser divergence. But the Hamamatsu R5509-73 NIR photomultiplier tube has a $3 \times 8 \mathrm{~mm}$ photocathode area size, resulting in a FOV of approx. $2 \mathrm{mrad}$.

A last important issue is the stability of the alignment. Especially in the case of long-range observations it is vital to have the laser and receiver accurately tuned at the target. Typical inertial measurement units (IMUs), responsible for direction stabilisation of airborne equipment, achieve accuracies of the order of $0.01-0.05^{\circ}$, or $0.2-1.0 \mathrm{mrad}$. Therefore, an LRP assembly with a much smaller angular resolution than $1 \mathrm{mrad}$ will typically not be able to achieve a stable lock on targets at large ranges.

The TNO LRP laser sends out ten pulses per second with a divergence of approx. 2 mrad. The detector, aligned with the laser, receives the reflection of the light from the same spot with a FOV of approx. $1.6 \mathrm{mrad}$. When a large target is illuminated relatively close to the sensor, the laser and detector require a scanning mechanism to cover the whole target. The range profile of each spot is recorded according to the time-of-flight principle. The final laser range profile for the target is obtained by adding up all individually recorded profiles. For example, the combined range profile for a target at 2.5 $\mathrm{km}$ distance with a 10 by $10 \mathrm{~m}$ surface area, and a detection FOV of approx. $4 \mathrm{~m}$ at that range, requires at least nine scans to cover the whole target (depending on the overlap of the individual FOVs). Figure 3.2 shows an example of this summation aspect.
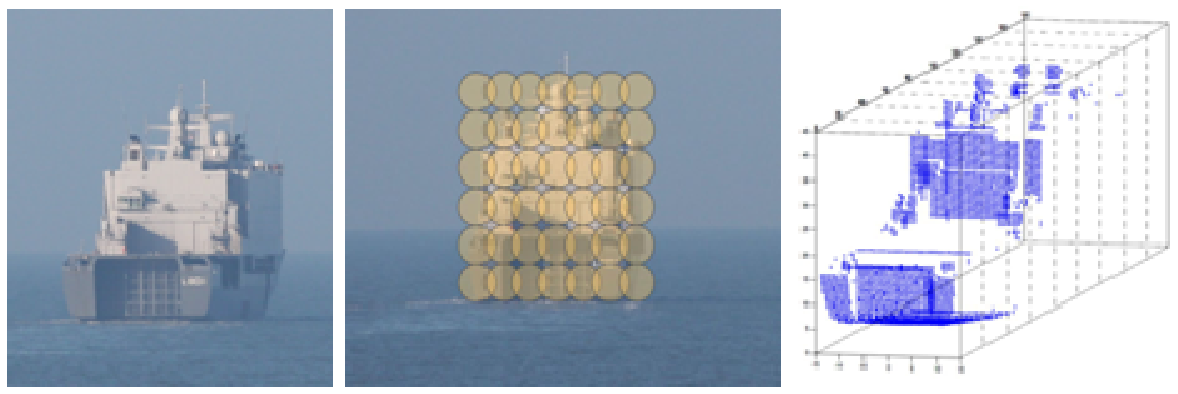

Figure 3.2: A Landing Platform Dock (LPD) of approx. $200 \mathrm{~m}$ length has been scanned at the back from approx. $4.2 \mathrm{~km}$ distance. To cover the whole target, 49 scans are required, see upper left and middle panel with zoom photos. The upper right panel shows the laser reflection points of the backside of the ship. Every circle in the middle panel is a profile that is to be combined into one end profile of the ship.

Because the scanning pattern is known in Figure 3.2, each recorded spot, having its own profile to be stored and analysed, contributes to a specific known part of the target. This spatial discrimination feature can be useful in cases were parts of targets need to be characterised.

It is also possible to use only one FOV to cover the whole target. If a target is relatively large, a dedicated lens with the right specifications and adjustments is required to expand the FOV. If, on the other hand, the target is small and at great distance, the standard lens FOV envelops the target completely and the reflected profile represents the whole target. This has advantages and disadvantages compared to the profile construction shown in Figure 3.2. The advantage is that the summation process, including its potential summation errors, can be skipped because only one profile is to be analysed. The disadvantage is the lack of information of each individual reflection, no spatial discrimination information is available for each individual spot. This is explained in Figure 3.3. Practically, the large FOV method should be sufficient for proper characterisation because most targets generate enough complex and unique features in the measured profile. 

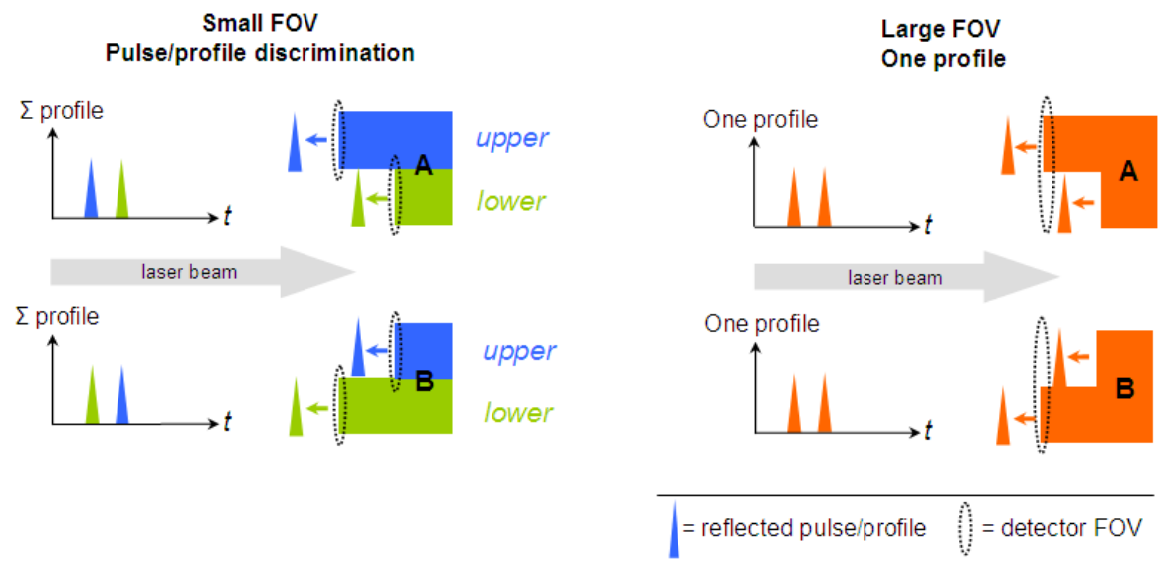

Figure 3.3: The difference between large and small FOV target profiling. Here two very simple targets A and B are represented as corner blocks. The orange target is identified as only one target, A or B. Keep in mind that the second (orange) measurement (B) could represent a, coincidentally, mirrored target A. The blue/green measurement with small FOV can easily distinguish between target A and B. If a target has sufficient high-resolution features that are asymmetrically distributed, however, the efficient low FOV technique suffices for adequate characterisation.

\subsection{Measurement campaign}

In April 2009 a 10-day field trial, with the name "NATO Maritime LADAR Target ID", was carried out in the harbour of San Diego, USA. The trial, falling under the NATO research group SET-132 that runs until the end of 2011, had three objectives for the data collection test. One of them was to collect a multi-mode database of 1D, 2D, and 3D laser detection and ranging (LADAR) imagery simultaneously with vibration sensing and passive (MWIR and Visible) imagery of maritime objects in such a way as to mimic multi-mode sensor scenarios in which MWIR and Visible sensors are used to search an area for detections of maritime objects and then transition to shorter range LADAR sensors to gather highresolution 1D, 2D, 3D imagery and vibration signatures for higher-level characterisation tasks such as identification. A total of 55 targets were identified during the trial. In this paper we used three of the 55 targets for a first testing of TNO's target characterisation efforts. The three civilian targets, with similar appearance, are depicted in Figure 3.4.
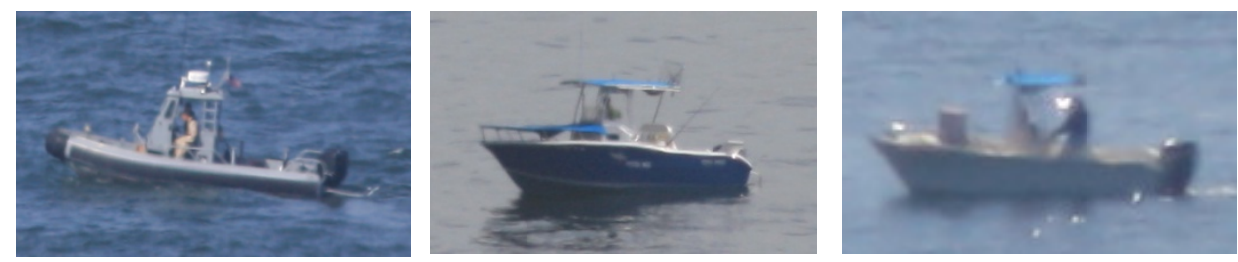

Figure 3.4: The physical targets, zoomed in. Left: A Rigid Hull Inflatable Boat (RHIB), designated as such, of $7 \mathrm{~m}$ length. A person is protected by a steel cage with a small round radar dome on top. Middle: A small private fishing boat of $5 \mathrm{~m}$ length, with solar protection roof, designated as FISH1. Right: A second small private fishing boat also with a solar protection roof yet with a cubic feature in front of the deck. This boat is of $6 \mathrm{~m}$ length and is designated as FISH2.

Common sensor systems have a hard job to recognise or identify small sea-surface craft at great distance. This is because of the target's length, height, and shape, which appear similar for most craft at ranges of $5 \mathrm{~km}$ and more. Nevertheless, identification of a small target at large distance becomes a daring technological achievement considering the range resolution of prototype laser range profiling systems. For example, the range resolution of the TNO LRP is $0.6 \mathrm{~m}$ which can be improved to 0.3 meters by way of deconvolution techniques, so single features in the centimetre scale are not distinguished, although small and more unique features - like antennas, beams, flags, fishing rods, wires, etc. - should in theory be distinguishable for the wavelengths of lasers. In any case, the a priori 3D modelling of small sea-surface targets should include the prominent distinguishable features for each type of vessel, so as to have a database that is diverse and large enough for every aspect angle and every feature to be considered. Characterisation is still possible if only distinct low-resolution features are present. This is shown in Figure 3.5, where crude models of the targets of Figure 3.4 display enough lo-res features for a proper characterisation effort with the resolution of the current LRP system in mind. 

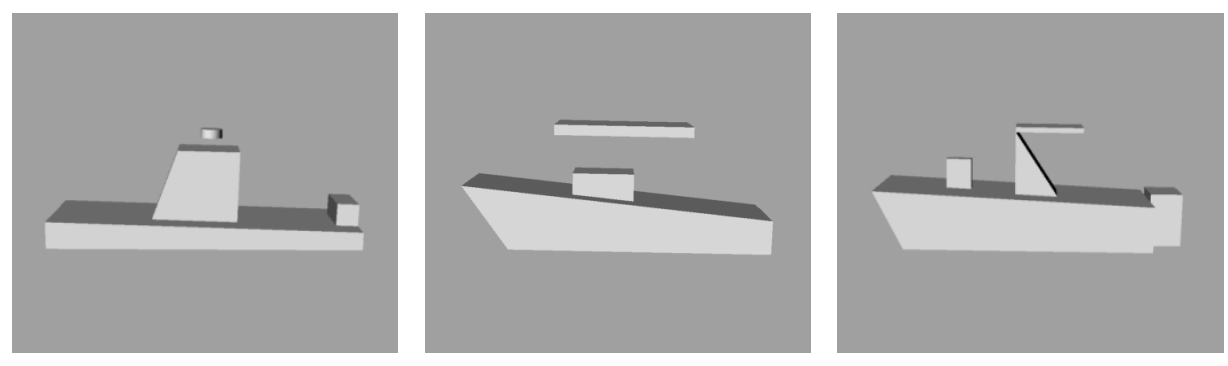

Figure 3.5: The crude-modelled targets according to Figure 3.5. Although the two fishing boats are very similar to each other (middle and right panel), low-resolution features can still be distinguished, providing a LRP characterisation opportunity.

\section{CHARACTERISATION OF SMALL TARGETS}

Target characterisation with range profiling techniques - be it categorisation, recognition or identification - can in general be separated into two groups: template-based characterisation, and feature-based characterisation. Template-based target characterisation is based on the comparison of the sample of interest (including all its features) with a database containing a (large) number of reference samples, each belonging to a certain class. A similarity measure is then used in order to decide to which class the given sample belongs. The database consists of range profiles for e.g. 72 aspect angles of the $3 \mathrm{D}$ modelled target, i.e. $5^{\circ}$ stepwise for $360^{\circ}$. This is for one plane only, and in case of sea targets this suffices, yet for air-to-air target characterisation the manoeuvrability requires a relatively huge database for every solid angle.

An alternative is feature-based target characterisation that uses low-dimensional features of the samples. The selected features should be maximally discriminative between different classes. A prominent part of feature-based target discrimination is the use of artificial neural networks.

\subsection{Characterisation methods}

LRPs are datasets which can be interpreted as histograms. The dissimilarity between two given histograms can be measured through bin-by-bin comparison or by cross-bin comparison. A combination of all pair wise comparisons can then be used as a measure of the distance between the two distributions. Target characterisation can then be performed by using, e.g., the Nearest Neighbour (NN) rule. In any case, in order to perform such a computation, a suitable distance has to be defined first. Several expressions for this distance or dissimilarity between histograms have been proposed. Eleven distances have been described in [5]. For this paper however we take three adequate methods:

- The Match Distance method, a promising candidate for efficient profiling. It is a cross-bin method that determines the distance between cumulative histograms with equal areas: $d_{M}(X, Y)=\sum_{i}\left|\hat{x}_{i}-\hat{y}_{i}\right|$ in which $\hat{x}_{i}=\sum_{j \leq i} x_{j}$

$X=\left\{x_{i}\right\}$ and $Y=\left\{y_{i}\right\}$ represent the histograms of interest and their elements. According to these definitions, the match distance is the distance between two cumulative histograms. Therefore, the weights of adjacent or rather preceding bins are taken into account in the comparison. However, the match distance cannot handle partial matches.

- The closely related Kolmogorov-Smirnov distance cross-bin method is also suited for LRP: $d_{K S}(X, Y)=\max _{i}\left(\left|\hat{x}_{i}-\hat{y}_{i}\right|\right)$, which is again defined using cumulative histograms. The $d_{K S}$ distance measure is commonly used in statistics for defining distances between non-binned distributions. In particular, the related Kolmogorov-Smirnov test is used to determine whether a given sample is drawn from a reference sample with a certain statistical distribution [6]. 
- The bin-by-bin Minkowski $L_{2}$ Distance method: $d_{L r}(X, Y)=\left(\sum_{i}\left|x_{i}-y_{i}\right|^{r}\right)^{1 / r}$. Several expressions for this distance or dissimilarity between histograms have been proposed. The Minkowski-form distance $d_{L r}$ is defined as [7,8]: $d_{L r}(X, Y)=\left(\sum_{i}\left|x_{i}-y_{i}\right|^{r}\right)^{1 / r}$, in which $\mathrm{X}=\left\{x_{i}\right\}$ and $\mathrm{Y}=\left\{y_{i}\right\}$ represent the histograms of interest and their elements. The order of the distance is given by $r$. Commonly used distances are the $L_{1}, L_{2}$, and $L_{\infty}$ distances. Other names for the $L_{l}$ and $L_{2}$ norms are 'city block norm' and 'Euclidean norm', respectively [9].

Simulated measurements were carried out using the 3D models of Figure 3.5. In order to emulate a real measurement, an incremental added noise was added to the simulations. So, a priori testing will show how a specific characterisation method performs. This is mostly needed because real measurements often suffer from inaccuracies like hardware adjustments, timing errors, environmental conditions, sparse datasets, or signal saturation, and combinations thereof. Several noise factors were used to see if the followed approach delivered the required results (Figure 4.1, continued on next page).
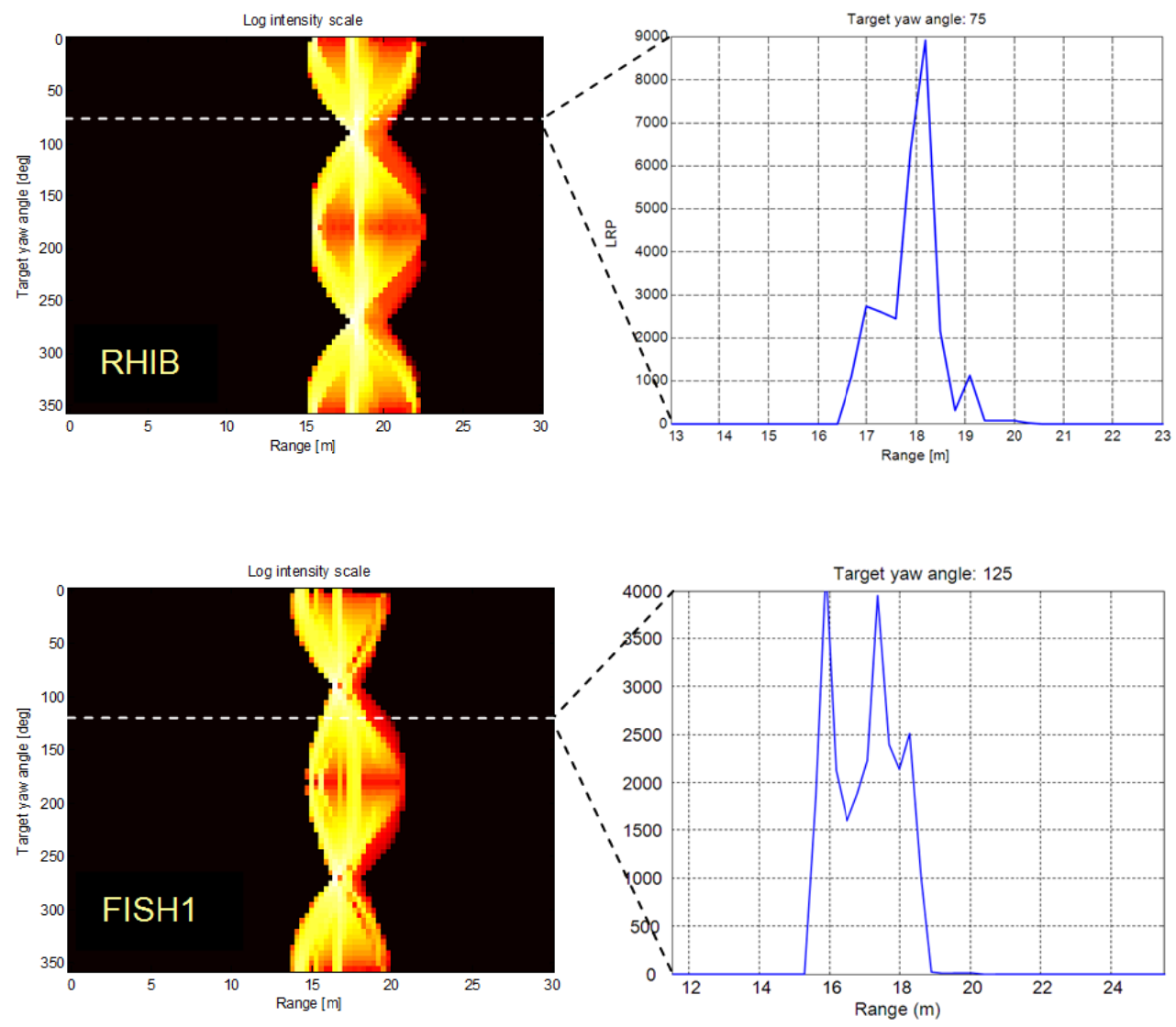

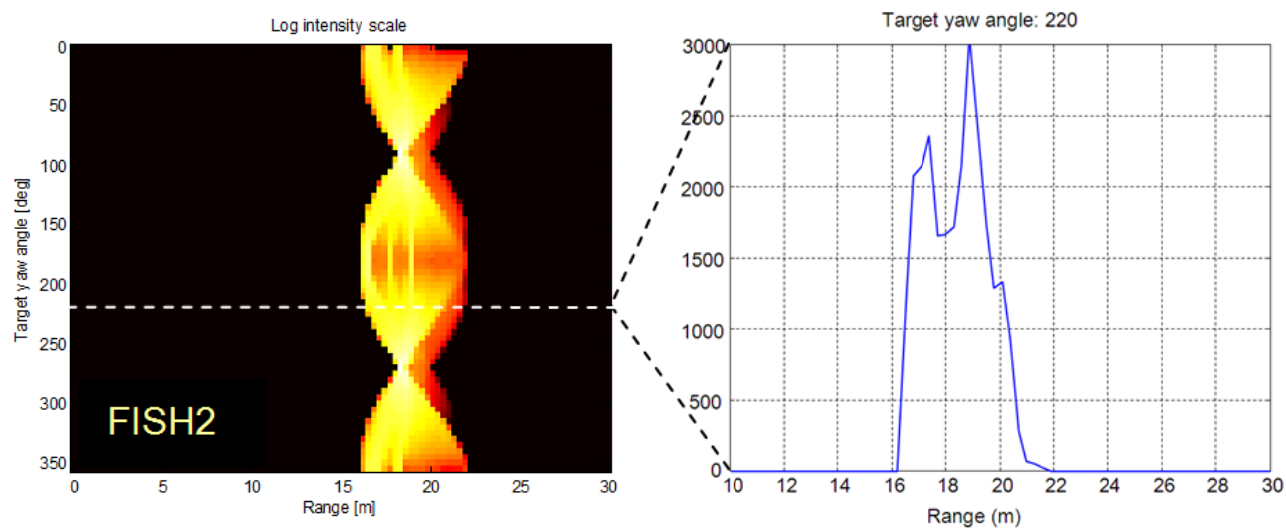

Figure 4.1: The simulated profiles per target for the database for all 72 aspect angles with $5^{\circ}$ steps (left). The panels on the right depict one laser range profile for one specific aspect angle (see cut in the left panels).

The real measurements delivered 19 good measurements for the RHIB and 28 good measurements for the FISH1 target. The FISH2 target provided only 6 measurements that were accurate enough for proper characterisation. The measurements incorporated only a few angles of attack. Three measured profiles from the experiment are shown in Figure 4.2.
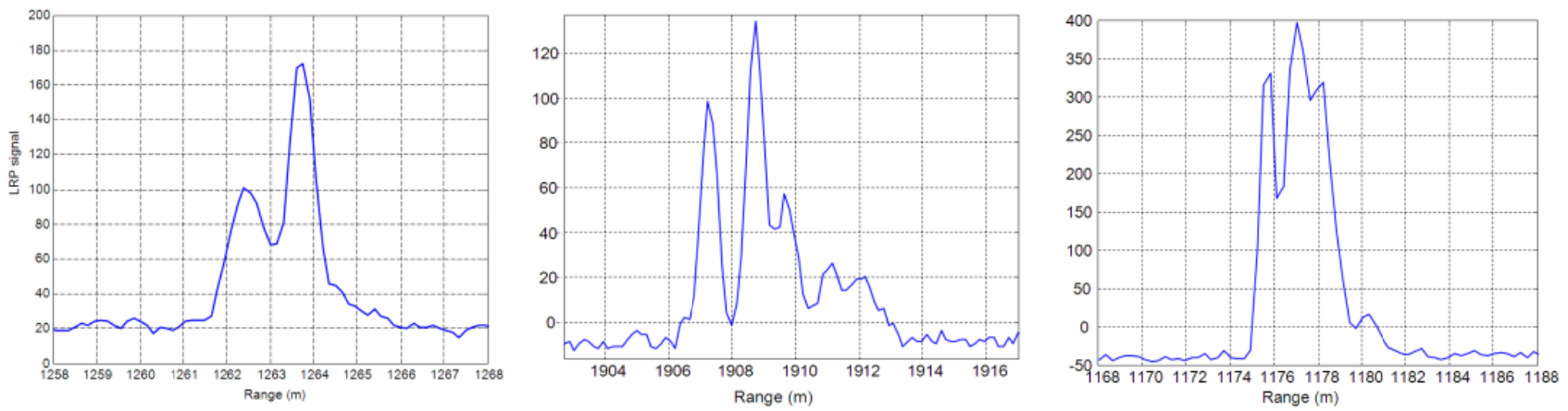

Figure 4.2: An LRP trial measured profile of each of the three targets at angles corresponding to the simulated profile cuts in Figure 6.5. The RHIB at range $1,282 \mathrm{~m}$ and aspect angle $75^{\circ}$ (left), FISH1 at range 1,906 $\mathrm{m}$ and an aspect angle of $125^{\circ}$ (middle), and the FISH2 at range $1,175 \mathrm{~m}$ and an aspect angle of $220^{\circ}$ (right).

By using the proper distance method, the class of the measured LRP will be defined by the class of the best-correlated LRP. Such a characterisation, in which the tested LRP is assigned to the class of its nearest neighbour in a set of training samples with known classes, is known as the Nearest Neighbour (NN) rule. An alternative method is the use of an arbitrarily high number of nearest neighbours. The best-suited class designation can then be selected on the basis of the largest number of proclaimed classes. This becomes important when we discuss the comparisons between the experiment and simulated results.

In order to assess the method used in the measurements, the balance of predicted data (a priori database targets) versus actual data (measurement data) is visualised by way of a confusion matrix. Confusion matrices originate from predictive analyses and give a reliability qualification of the numerical characterisation method used in relation to the specific dataset. We start by showing the simulated results for five noise increments and up to five nearest neighbours in the next subsection.

\subsection{Simulated characterisation results}

The target characterisation confusion matrices for the simulated profiles are shown in Table 4.1, followed by a discussion.

Table 4.1. Confusion matrices for the simulated measurements performed with the Match Distance method. Five noise levels and up to five nearest neighbours $(\mathrm{NN})$ have been computed. 


\begin{tabular}{|c|c|c|c|c|c|c|c|c|c|c|}
\hline \multirow{3}{*}{\multicolumn{2}{|c|}{$\mathrm{NN}=1$}} & \multicolumn{3}{|c|}{ Noise sigma $=0$} & \multicolumn{3}{|c|}{ Noise sigma $=100$} & \multicolumn{3}{|c|}{ Noise sigma $=200$} \\
\hline & & \multicolumn{3}{|c|}{ Predicted } & \multicolumn{3}{|c|}{ Predicted } & \multicolumn{3}{|c|}{ Predicted } \\
\hline & & RHIB & FISH 1 & $\mathrm{FISH} 2$ & RHIB & FISH 1 & FISH2 & RHIB & FISH 1 & FISH2 \\
\hline \multirow{3}{*}{ 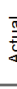 } & RHIB & 1.00 & 0.00 & 0.00 & 0.99 & 0.00 & 0.01 & 0.94 & 0.02 & 0.04 \\
\hline & FISH1 & 0.00 & 1.00 & 0.00 & 0.01 & 0.93 & 0.06 & 0.08 & 0.73 & 0.19 \\
\hline & $\mathrm{FISH} 2$ & 0.00 & 0.00 & 1.00 & 0.00 & 0.04 & 0.96 & 0.04 & 0.13 & 0.83 \\
\hline \multicolumn{2}{|r|}{$\mathrm{NN}=\mathbf{2}$} & RHIB & FISH1 & $\mathrm{FISH} 2$ & RHIB & FISH1 & FISH2 & RHIB & FISH1 & FISH2 \\
\hline \multirow{3}{*}{$\begin{array}{l}\bar{a} \\
\overline{4}\end{array}$} & RHIB & 1.00 & 0.00 & 0.00 & 1.00 & 0.00 & 0.00 & 0.99 & 0.00 & 0.01 \\
\hline & FISH1 & 0.00 & 1.00 & 0.00 & 0.01 & 0.96 & 0.03 & 0.09 & 0.79 & 0.12 \\
\hline & FISH2 & 0.00 & 0.00 & 1.00 & 0.00 & 0.01 & 0.99 & 0.04 & 0.15 & 0.81 \\
\hline \multicolumn{2}{|r|}{$\mathrm{NN}=3$} & RHIB & $\mathrm{F} \mid \mathrm{SH} 1$ & $\mathrm{FISH} 2$ & RHIB & $\mathrm{FISH} 1$ & $\mathrm{FISH} 2$ & RHIB & $\mathrm{FISH} 1$ & $\mathrm{FISH} 2$ \\
\hline \multirow{3}{*}{ ब, } & RHIB & 0.90 & 0.00 & 0.10 & 0.98 & 0.01 & 0.01 & 0.97 & 0.02 & 0.01 \\
\hline & FISH1 & 0.00 & 0.87 & 0.13 & 0.01 & 0.88 & 0.11 & 0.07 & 0.74 & 0.19 \\
\hline & $\mathrm{FISH} 2$ & 0.00 & 0.62 & 0.38 & 0.00 & 0.58 & 0.42 & 0.02 & 0.51 & 0.47 \\
\hline \multicolumn{2}{|r|}{$\mathrm{NN}=4$} & RHIB & FISH1 & $\mathrm{FISH} 2$ & RHIB & FISH1 & FISH2 & RHIB & FISH1 & FISH2 \\
\hline \multirow{3}{*}{ } & RHIB & 0.60 & 0.26 & 0.14 & 0.64 & 0.28 & 0.08 & 0.70 & 0.23 & 0.07 \\
\hline & FISH1 & 0.00 & 0.95 & 0.05 & 0.01 & 0.94 & 0.05 & 0.09 & 0.79 & 0.12 \\
\hline & $\mathrm{FISH} 2$ & 0.00 & 0.77 & 0.23 & 0.01 & 0.74 & 0.25 & 0.06 & 0.65 & 0.29 \\
\hline \multicolumn{2}{|r|}{$\mathrm{NN}=5$} & RHIB & FISH1 & $\mathrm{FISH} 2$ & RHIB & FISH1 & FISH2 & RHIB & FISH1 & FISH2 \\
\hline \multirow{3}{*}{ 商 } & RHIB & 0.86 & 0.14 & 0.00 & 0.80 & 0.16 & 0.04 & 0.79 & 0.15 & 0.06 \\
\hline & FISH1 & 0.00 & 0.88 & 0.12 & 0.01 & 0.89 & 0.10 & 0.09 & 0.75 & 0.16 \\
\hline & FISH2 & 0.00 & 0.63 & 0.37 & 0.00 & 0.63 & 0.37 & 0.02 & 0.54 & 0.44 \\
\hline
\end{tabular}

\begin{tabular}{|c|c|c|c|c|c|c|c|c|c|c|}
\hline \multirow{3}{*}{\multicolumn{2}{|c|}{$N N=1$}} & \multicolumn{3}{|c|}{ Noise sigma $=300$} & \multicolumn{3}{|c|}{ Noise sigma $=400$} & \multicolumn{3}{|c|}{ Noise sigma $=500$} \\
\hline & & \multicolumn{3}{|c|}{ Predicted } & \multicolumn{3}{|c|}{ Predicted } & \multicolumn{3}{|c|}{ Predicted } \\
\hline & & RHIB & FISH1 & $\mathrm{FISH} 2$ & RHIB & FISH1 & FISH2 & RHIB & FISH1 & $\mathrm{FISH} 2$ \\
\hline \multirow{3}{*}{ 밍. } & RHIB & 0.87 & 0.06 & 0.07 & 0.81 & 0.09 & 0.10 & 0.81 & 0.07 & 0.12 \\
\hline & FISH1 & 0.10 & 0.65 & 0.25 & 0.18 & 0.53 & 0.29 & 0.28 & 0.43 & 0.29 \\
\hline & FISH2 & 0.11 & 0.20 & 0.69 & 0.15 & 0.23 & 0.62 & 0.18 & 0.25 & 0.57 \\
\hline \multicolumn{2}{|r|}{$\mathrm{NN}=2$} & RHIB & FISH1 & FISH2 & RHIB & FISH1 & FISH2 & RHIB & FISH1 & $\mathrm{FISH} 2$ \\
\hline \multirow{3}{*}{$\overline{\frac{a}{3}}$} & $\mathrm{RHIB}$ & 0.98 & 0.02 & 0.00 & 0.88 & 0.06 & 0.06 & 0.88 & 0.06 & 0.06 \\
\hline & FISH1 & 0.15 & 0.65 & 0.20 & 0.30 & 0.55 & 0.15 & 0.43 & 0.36 & 0.21 \\
\hline & $\mathrm{FISH} 2$ & 0.17 & 0.16 & 0.67 & 0.26 & 0.20 & 0.54 & 0.33 & 0.19 & 0.48 \\
\hline \multicolumn{2}{|r|}{$\mathrm{NN}=3$} & RHIB & FISH1 & $\mathrm{FISH} 2$ & RHIB & $\mathrm{FISH} 1$ & $\mathrm{FISH} 2$ & RHIB & $\mathrm{FISH} 1$ & $\mathrm{FISH} 2$ \\
\hline \multirow{3}{*}{$\bar{\Xi}$} & RHIB & 0.91 & 0.05 & 0.04 & 0.82 & 0.10 & 0.08 & 0.85 & 0.09 & 0.06 \\
\hline & FISH1 & 0.10 & 0.66 & 0.24 & 0.20 & 0.59 & 0.21 & 0.28 & 0.51 & 0.21 \\
\hline & $\mathrm{FISH} 2$ & 0.09 & 0.43 & 0.48 & 0.18 & 0.45 & 0.37 & 0.19 & 0.40 & 0.41 \\
\hline \multicolumn{2}{|r|}{$\mathrm{NN}=4$} & RHIB & FISH1 & $\mathrm{FISH} 2$ & RHIB & FISH1 & FISH2 & RHIB & FISH1 & FISH2 \\
\hline \multirow{3}{*}{$\bar{\Xi}$} & RHIB & 0.72 & 0.20 & 0.08 & 0.69 & 0.22 & 0.09 & 0.70 & 0.21 & 0.09 \\
\hline & FISH1 & 0.10 & 0.71 & 0.19 & 0.22 & 0.64 & 0.14 & 0.29 & 0.53 & 0.18 \\
\hline & $\mathrm{FISH} 2$ & 0.12 & 0.57 & 0.31 & 0.17 & 0.52 & 0.31 & 0.20 & 0.51 & 0.29 \\
\hline \multicolumn{2}{|r|}{$\mathrm{NN}=5$} & RHIB & FISH1 & $\mathrm{FISH} 2$ & RHIB & FISH1 & FISH2 & RHIB & FISH1 & FISH2 \\
\hline & RHIB & 0.79 & 0.14 & 0.07 & 0.74 & 0.14 & 0.12 & 0.77 & 0.16 & 0.07 \\
\hline & FISH1 & 0.10 & 0.67 & 0.23 & 0.22 & 0.60 & 0.18 & 0.30 & 0.49 & 0.21 \\
\hline & FISH2 & 0.09 & 0.50 & 0.41 & 0.13 & 0.44 & 0.43 & 0.20 & 0.40 & 0.40 \\
\hline
\end{tabular}

The results in Table 4.1 show that using more than one nearest neighbour does not necessarily contribute to a better confusion matrix. It seems that unpredictable fluctuations in the values show up for different values of NN. This inconsistency is observed for the noiseless case as well as for the simulations with added noise. On the other hand, for the $\mathrm{NN}=$ 1 case, when more noise is added, more confusion is present in the prediction. This makes us wonder how to interpret the use of nearest neighbours in this simulation. The database has profiles for each target for 72 aspect angles of the $360^{\circ}$ view. The angular resolution is $5^{\circ}$. The $3 \mathrm{D}$ models were modelled with low-resolution because the range resolution is 0.6 meter (Subsection 3.2), while they show enough low-resolution features for proper characterisation. That was the idea. What this actually implies is when the aspect angle is turned one step $\left(5^{\circ}\right)$, the target can be predicted as the other target. The angular resolution appears to be too coarse for the low-resolution details to be distinctive enough when using more than one nearest neighbour. Thus for the experimental profiling efforts done in San Diego only one nearest neighbour must be used for a consequent and accurate qualification.

\subsection{Experimental characterisation results}

In Subsection 4.1 it was stipulated to investigate the three most promising numerical methods for laser range profiling, i.e. the Match Distance method, the Kolmogorov-Smirnov Distance method, and the Minkowski $L_{2}$ Distance method. The confusion matrices of the simulated measurements revealed that using more than one nearest neighbour will not result in a better characterisation for the current system and angular resolution used. Therefore, in Table 4.2 the confusion matrices for one nearest neighbour is shown for the three appropriate distances.

Table 4.2. Confusion matrices for the experimental measurements for the first nearest neighbour.

\begin{tabular}{|c|c|c|c|c|c|c|c|c|c|c|}
\hline & \multicolumn{3}{|c|}{ Match Distance } & \multicolumn{3}{|c|}{ Kolmogorov-Smirnov } & \multicolumn{3}{|c|}{ Minkowski $L_{2}$} \\
\hline & & \multicolumn{3}{|c|}{ Predicted } & \multicolumn{3}{|c|}{ Predicted } & \multicolumn{3}{|c|}{ Predicted } \\
\hline & $\mathrm{NN}=1$ & RHIB & $\mathrm{FISH} 1$ & $\mathrm{FISH} 2$ & RHIB & FISH1 & $\mathrm{FISH} 2$ & RHIB & FISH1 & $\mathrm{FISH} 2$ \\
\hline \multirow{3}{*}{$\begin{array}{l}\bar{\pi} \\
\frac{0}{0} \\
\end{array}$} & RHIB & 0.58 & 0.16 & 0.26 & 0.68 & 0.16 & 0.16 & 0.58 & 0.16 & 0.26 \\
\hline & $\mathrm{FISH} 1$ & 0.18 & 0.36 & 0.46 & 0.18 & 0.43 & 0.39 & 0.14 & 0.32 & 0.54 \\
\hline & $\mathrm{FISH} 2$ & 0.67 & 0.00 & 0.33 & 0.50 & 0.33 & 0.17 & 0.67 & 0.00 & 0.33 \\
\hline
\end{tabular}

Obviously, the results for these three targets are quite different when compared to Table 4.1. The Match Distance matrix does not even come close to the most noisy $\mathrm{NN}=1$ matrix in Table 4.1. This also holds for the other two methods. Moreover, the distribution in each matrix does not correspond to those in Table 4.1. Note the FISH2 outlier, i.e. FISH2 is wrongly predicted as a RHIB by all three methods $(0.67,0.50,0.67)$, whereas the Kolmogorov-Smirnov has at least a less wrong FISH1 prediction (0.33) included. Several causes are identified. First and foremost, the dataset for FISH2 with only six samples is too sparse. Second, the angular resolution in the database, which is too coarse for comparisons with profile measurements at 0.6 meter range resolution. And third, the inaccuracies due to practical limitations, as mentioned earlier, have a big influence on the final outcomes. Clearly, a desired $95 \%$ accuracy (close to the $\mathrm{NN}=1$, noise sigma $=100$ simulation) has not been obtained. 


\section{CONCLUSIONS}

The projected surface area of the targets in this paper lies between 10 and $15 \mathrm{~m}^{2}$, which is too small for proper laser range profiling identification using a range resolution of $60 \mathrm{~cm}$ as per the outcomes in Subsection 4.3. The targets were at $>1 \mathrm{~km}$ range, but range is not leading, contrary to the ratio between a) the laser range resolution and b) the size of the targets and the size of distinguishable features of the targets. This ratio, prescribing a successful profiling, seems too high in the analysis.

Next to the discussions on the outcomes in Subsections 4.2 and 4.3, the questions posed in Section 1, the introduction, which define a kind of basis of the paper, can now be answered as follows:

- Which numerical methods are suitable for target identification?

In Subsection 4.1, where an analysis of range profiling techniques is presented, three one-dimensional distance methods have been identified that are efficient, accurate, and promising enough for future operational characterisation efforts. These methods are the Match Distance Method, the Kolmogorov - Smirnov Distance Method, and the Minkowski $L_{2}$ Distance Method, and were used in efforts to characterise small targets from experimental data as presented in this paper.

- Are 3D models convenient to generate a database for laser range profiling?

The use of low-resolution 3D models for template-based characterisation of laser range profiling targets happens to be an adequate and efficient way. Low-resolution 3D models can be quickly made, which is convenient when new targets are required for an operational database. High-resolution 3D models obviously take longer time to construct, but also come with similar or better results than low-resolution models. Low-resolution boat model tests are presented in Subsection 3.2 where the measurement is shortly discussed. The reasons to use the low-resolution model in this research are twofold. Firstly, the range resolution of the used laser range profiler is relatively low $(0.6 \mathrm{~m})$, and secondly, it is less labour intensive to construct 3D models at lower resolution. Unfortunately, this approach delivers suboptimal characterisation results if the database is too sparse and the quality of the data suffers from external influences. This is presented and discussed in Subsection 4.3.

- Which range resolution is feasible for target characterisation and which targets are identifiable for a certain resolution?

While the range resolution of TNO's LRP system is $60 \mathrm{~cm}$, which could be halved using deconvolution techniques, stateof-the-art systems however should be able to achieve a $5-10 \mathrm{~cm}$ range resolution. In another work [5], two feasibility studies were performed considering the range performance of current and state-of-the-art LRP systems, resulting in several findings. The atmosphere (visibility) between laser and target and the size of the target play an important role for range performance. Large targets of approx. $1000 \mathrm{~m}^{2}$ (e.g. sea cargo vessels) can be accurately characterised at approx. $17 \mathrm{~km}$ with TNO's LRP system under perfect weather conditions. With a state-of-the-art system this range is extended to $100-120 \mathrm{~km}$. For small targets of $50 \mathrm{~m}^{2}$ (e.g. airplanes), the characterisation range is $8-10 \mathrm{~km}$ for TNO's system, while for a state-of-the-art system the range runs from 75 to $160 \mathrm{~km}$, where $75 \mathrm{~km}$ applies to a visibility of $30 \mathrm{~km}$ and $160 \mathrm{~km}$ represents free space with no attenuation due to molecules and aerosols. For targets of $50 \mathrm{~m}^{2}$ and bigger, a range resolution of $30-60 \mathrm{~cm}$ is in principle sufficient for target identification (see e.g. Figure 3.2).

Next to these answers, some additional remarks are put forward.

In Subsections 4.1 and 4.2 the confusion matrix and its function was shortly discussed within the context of the small sea-surface target characterisation experiment. With the results and the knowledge gained so far, the importance of a confusion matrix of good quality is noticed. The confusion matrix must be a reliable source of information if an operational LRP system is to be used in a scenario with unknown targets. The characterisation method must be validated on its accuracy and reliability. An a priori database consists of a certain class or classes of targets and is used to characterise these targets in a scenario. Unknown targets are to be categorised as 'other'. In the confusion matrix this 'known - other' discrimination plus the correct prediction for all (a priori) known targets should be performed with a preferred accuracy of $100 \%$. Depending on the user requirements, however, $95 \%$ is mostly seen as the lower limit for operational applications. Besides, the more extensive the database is the fewer unknowns have to be characterised. The confusion matrix thus is essential as a reliability test for software to be developed for operational use.

Targets are usually not of the same size in operational settings. Laser range profiling measurements were performed on large targets in previous projects, while in this report the only actors were small targets. For laser range profiling the 
target size, the range, and the detector FOV are all of importance. Future operational laser range profiler systems could, however, be equipped with discriminative lens/FOV systems or with a system that is flexible enough for switching between small and large targets at different ranges. The latter option is feasible with the current technology at hand.

\section{ACKNOWLEDGEMENTS}

We thank the Netherlands Ministry of Defence and especially the Defence Materiel Organisation for supporting this work. Special thanks go to Lieutenant Menno Smeelen of the Royal Netherlands Navy for stimulating discussions. We also thank Bob Hintz, chairman of the NATO SET-132 TG-72 group, and his team, who arranged the 2009 measurement campaign in San Diego.

\section{REFERENCES}

[1] J.C. van den Heuvel, 'Eindrapportage programma Laser Applicaties V404', TNO-DV 2007 A420.

[2] J.C. van den Heuvel, H.H.P.Th Bekman, F.J.M van Putten, and L.H. Cohen, 'Identification of littoral targets with a laser range profiler' Proc. SPIE 6550, 65500R (2007).

[3] J.C. van den Heuvel, P. Pace, H.H.P.Th. Bekman, F.J.M. van Putten, and R.M.A. Schleijpen, 'Experimental validation of ship identification with a laser range profiler', Proc. SPIE 6950, 69500V (2008).

[4] 'Relative Performance of Correlation-Based and Feature-Based Classifiers of Aircraft Using Radar Range Profiles', C. Nieuwoudt, E.C. Botha, 4th Int. Conf. on Pattern Recognition (ICPR'98), Vol 2, 1998, pp.1828.

[5] R.M. Schoemaker, K.W. Benoist, G.C. Franssen, 'Laser Range Profiling', TNO-DV 2010 IN375.

[6] NIST/SEMATECH e-Handbook of Statistical Methods, http://www.itl.nist.gov/div898/handbook/eda/section3/eda35g.htm

[7] Y. Rubner, C. Tomasi, and L.J. Guibas, 'The earth mover's distance as a metric for image retrieval', Int. J. Comput. Vision 40, 99 (2000).

[8] M. Stricker and M. Orengo, 'Similarity of color images', Proc. SPIE 2420, 381 (1995).

[9] S.-H. Cha and S.N. Srihari, 'On measuring the distance between histograms', Pattern Recogn. 35, 1355 (2002). 